\title{
From coupled Rashba electron- and hole-gas layers to three-dimensional topological insulators
}

\author{
Luka Trifunovic, ${ }^{1,2}$ Daniel Loss, ${ }^{1}$ and Jelena Klinovaja ${ }^{1}$ \\ ${ }^{1}$ Department of Physics, University of Basel, Klingelbergstrasse 82, CH-4056 Basel, Switzerland \\ ${ }^{2}$ Dahlem Center for Complex Quantum Systems and Physics Department, Freie Universität Berlin, Arnimallee 14, 14195 Berlin, Germany
}

(Received 5 November 2015; published 4 May 2016)

\begin{abstract}
We introduce a system of stacked two-dimensional electron- and hole-gas layers with Rashba spin-orbit interaction and show that the tunnel coupling between the layers induces a strong three-dimensional (3D) topological insulator phase. At each of the two-dimensional bulk boundaries we find the spectrum consisting of a single anisotropic Dirac cone, which we show by analytical and numerical calculations. Our setup has a unit cell consisting of four tunnel coupled Rashba layers and presents a synthetic strong 3D topological insulator and is distinguished by its rather high experimental feasibility.
\end{abstract}

DOI: 10.1103/PhysRevB.93.205406

\section{INTRODUCTION}

Since the discovery of the quantum Hall effect there has been immense theoretical interest focused on understanding topological phases of quantum matter [1,2]. The interest has not been concentrated solely on the classification of these novel phases, [3] which goes beyond the Landau paradigm of phase transitions, but also on the potential applications of the topologically ordered phases, in particular for storing quantum information in a manner that is resilient to local imperfections [4]. Additionally, the electronic surface states of a strong topological insulator (TI) [2,5], being an example of a three-dimensional (3D) topological phase of matter, forms a two-dimensional (2D) topological metal, which is "half" of an ordinary metal [2]. Such 2D topological metals are notable for the fact that their electrons cannot be localized even in the presence of strong disorder, as long as the bulk energy gap of the parent strong 3D TI is intact [6].

There are strong indications that certain materials, such as semiconducting alloys, behave as strong 3D TIs [2]. Despite great success in this field, both theoretically and experimentally, there are still certain issues that need to be resolved, in particular that strong TIs suffer from bulk conduction due to chemical imperfections. Thus, there is a strong need for synthetic materials where one has enough control over the system parameters in order to achieve a topological phase with a sufficiently large bulk gap which excludes bulk conduction.

One of the very successful approaches for theoretically constructing 2D topological phases of matter is using anisotropic hopping or a coupled wire construction [7-21]. Apart from being very intuitive, this approach allows nonperturbative treatment of the electron-electron interactions and is thus suitable for the study of fractional topological phases. Recently, a strong effort was made to extend this approach to the study of 3D TIs, where topological phases related to weak TIs were obtained, as well as Weyl semimetal phases [21-23]. Despite the great theoretical insight this approach gives, its main drawback in the case of 3D systems is that the resulting setups are rather complex and thus not easy to realize experimentally.

In this paper we take a different approach, instead of coupled wires [7-22] we introduce a construction of coupled 2D layers, see Fig. 1. Each layer is a simple 2D electron gas (2DEG) with Rashba spin-orbit interaction (SOI) [24-27].
By generalizing the coupled wires approach [17] to coupled layers we arrive at a rather simple realization of a strong 3D TI. Inclusion of electron-electron interactions in 2D systems is much more involved than the 1D case, but still possible [28]. We note in passing that there are some proposals for realization of synthetic strong TIs in ultracold atoms systems [29].

\section{MODEL}

We consider a system consisting of tunnel coupled layers of 2DEGs stacked along the $z$ axis, see Fig. 1. In each 2DEG we include an SOI [25] and we assume it to be of Rashba type. ${ }^{1}$ In our model, we work with two different values of SOI [30-32] that could be chosen almost arbitrarily (see below) and do not require special tuning. In contrast to that, the chemical potential $\mu_{\tau}$ in each layer should be individually tuned to the value determined by the corresponding SOI. Our setup has a unit cell consisting of four Rashba 2DEG layers.

A single 2DEG layer with Rashba SOI is described by the Hamiltonian [24]

$$
H_{0}=-\hbar^{2}\left(\partial_{x}^{2}+\partial_{y}^{2}\right) / 2 m_{0}-i \alpha\left(\sigma^{x} \partial_{y}-\sigma^{y} \partial_{x}\right),
$$

where $\alpha$ is the strength of the Rashba SOI and $m_{0}$ the electron mass in the given band. We can diagonalize this Hamiltonian by taking the local spin quantization axis $s=(-\sin \theta, \cos \theta)$ to be always perpendicular to the momentum $\boldsymbol{k}=\left(k_{x}, k_{y}\right) \equiv$ $k(\cos \theta, \sin \theta)$,

$$
E_{\mp}(\boldsymbol{k})=\hbar^{2} k^{2} / 2 m_{0} \mp \alpha k,
$$

where the upper (lower) sign corresponds to the spin orientation being along (opposite to) $s$ chosen for $\alpha>0$ and to the lower (higher) energy for a fixed $\boldsymbol{k}$, where the corresponding spinors are given by

$$
|\mp ; \theta\rangle=\frac{1}{\sqrt{2}}\left(\begin{array}{c}
1 \\
\pm i e^{i \theta}
\end{array}\right) .
$$

We note here that the spin orientation is clockwise (anticlockwise) for $|+; \theta\rangle(|-; \theta\rangle)$. The dispersion relation Eq. (2) is

\footnotetext{
${ }^{1}$ Our results still hold if Rashba is replaced by Dresselhaus SOIs. When both Rashba and Dresselhaus SOIs are present, our scheme still works if one of them dominates.
} 
(a)

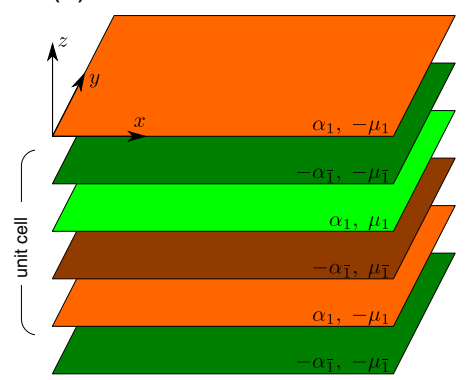

(b)

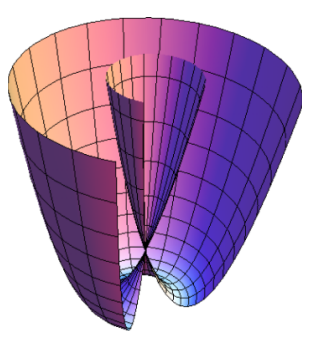

FIG. 1. (a) Setup consisting of a stack of layers arranged in the $x y$ plane and tunnel coupled along the $z$ axis. The layers colored in green (orange) denote electron (hole) 2DEGs with Rashba SOI and at chemical potential $\mu_{\tau}$. The brightness of the color encodes two possible values of the Rashba SOI $\alpha_{\tau}$. (b) Dispersion of a 2DEG with Rashba SOI.

depicted in Fig. 1(b), and the shape of the Fermi surfaces and the spin orientations in Fig. 2(b).

The setup we consider herein consists of four stacked layers composing the unit cell, which then periodically repeats in the $z$ direction with spacing $a_{z}$ between layers. Each of the four layers of the unit cell is labeled by two indices $\eta= \pm 1$ and $\tau= \pm 1$. The index $\eta=1(\eta=\overline{1})$ corresponds to an electron (hole) dispersion relation. The index $\tau$ refers to two different values of the SOI, $\alpha_{1}$ and $\alpha_{\overline{1}}$, where without loss of generality we assume that $0<\alpha_{1}<\alpha_{\overline{1}}$. The ordering of the layers inside the unit cell is shown in Fig. 2(a). Two electron layers are followed by two hole layers as the SOI magnitude $\alpha_{\tau}$ alternates from layer to layer.

The total Hamiltonian of the system is $H=$ $\sum_{n=1}^{N} \int d x d y \mathcal{H}_{n}(x, y)$, where $N$ is the total number of unit cells and the Hamiltonian density is given by $\mathcal{H}_{n}=\mathcal{H}_{n 0}+\mathcal{H}_{n t}$ with $\mathcal{H}_{n 0}=\sum_{\{\tau, \eta=1, \overline{1}\}} \mathcal{H}_{n \eta \tau}$, where

$$
\begin{aligned}
\mathcal{H}_{n \eta \tau}= & \sum_{\sigma, \sigma^{\prime}} \Psi_{n \eta \tau \sigma}^{\dagger}\left[-\frac{\eta \hbar^{2}}{2 m_{0}}\left(\partial_{x}^{2}+\partial_{y}^{2}\right)+\eta \tau \mu_{\tau}\right. \\
& \left.-i \tau \alpha_{\tau}\left(\sigma^{x} \partial_{y}-\sigma^{y} \partial_{x}\right)\right]_{\sigma \sigma^{\prime}} \Psi_{n \eta \tau \sigma^{\prime}}
\end{aligned}
$$

The electron (hole) annihilation operator $\Psi_{n \eta \tau \sigma}(\boldsymbol{r})$ acts on particles with spin $\sigma$ at the position $\boldsymbol{r}=(x, y)$ of the $(n \eta \tau)$ layer. The chemical potential $\mu_{\tau}$ is calculated from the crossing point at $k=0$ determined by the SOI energy $E_{\mathrm{So}, \tau}=\hbar^{2} k_{\mathrm{so}, \tau}^{2} / 2 m_{0}$ with the SOI wave vector $k_{\mathrm{so}, \tau}=m_{0} \alpha_{\tau} / \hbar^{2}$. The dispersion relation (for fixed $\theta$ ) of each layer is shown in Fig. 2(a) and can be easily generalized to all directions of $\boldsymbol{k}$. In the following, we fix the chemical potentials as $\mu_{1}=\mu_{\overline{1}}=E_{\mathrm{so}, \overline{1}}-E_{\mathrm{so}, 1}$. This choice ensures that the interior (exterior) Fermi surfaces have the same radius $k_{F i}=k_{\mathrm{so}, \overline{1}}-k_{\mathrm{so}, 1}\left(k_{F e}=k_{\mathrm{so}, \overline{1}}+k_{\mathrm{so}, 1}\right)$ across all the layers. Additionally, we need to assume that $\mu_{\tau} \gg t$.

The tunneling between the layers is assumed to be spin independent and takes the following form:

$$
\mathcal{H}_{n t}=\sum_{\sigma\left\langle\tau \eta ; n^{\prime} \tau^{\prime} \eta^{\prime}\right\rangle} t \Psi_{n \eta \tau \sigma}^{\dagger}(\boldsymbol{r}) \Psi_{n^{\prime} \eta^{\prime} \tau^{\prime} \sigma}(\boldsymbol{r})+\text { H.c. },
$$

where the summation runs over all neighboring layers.

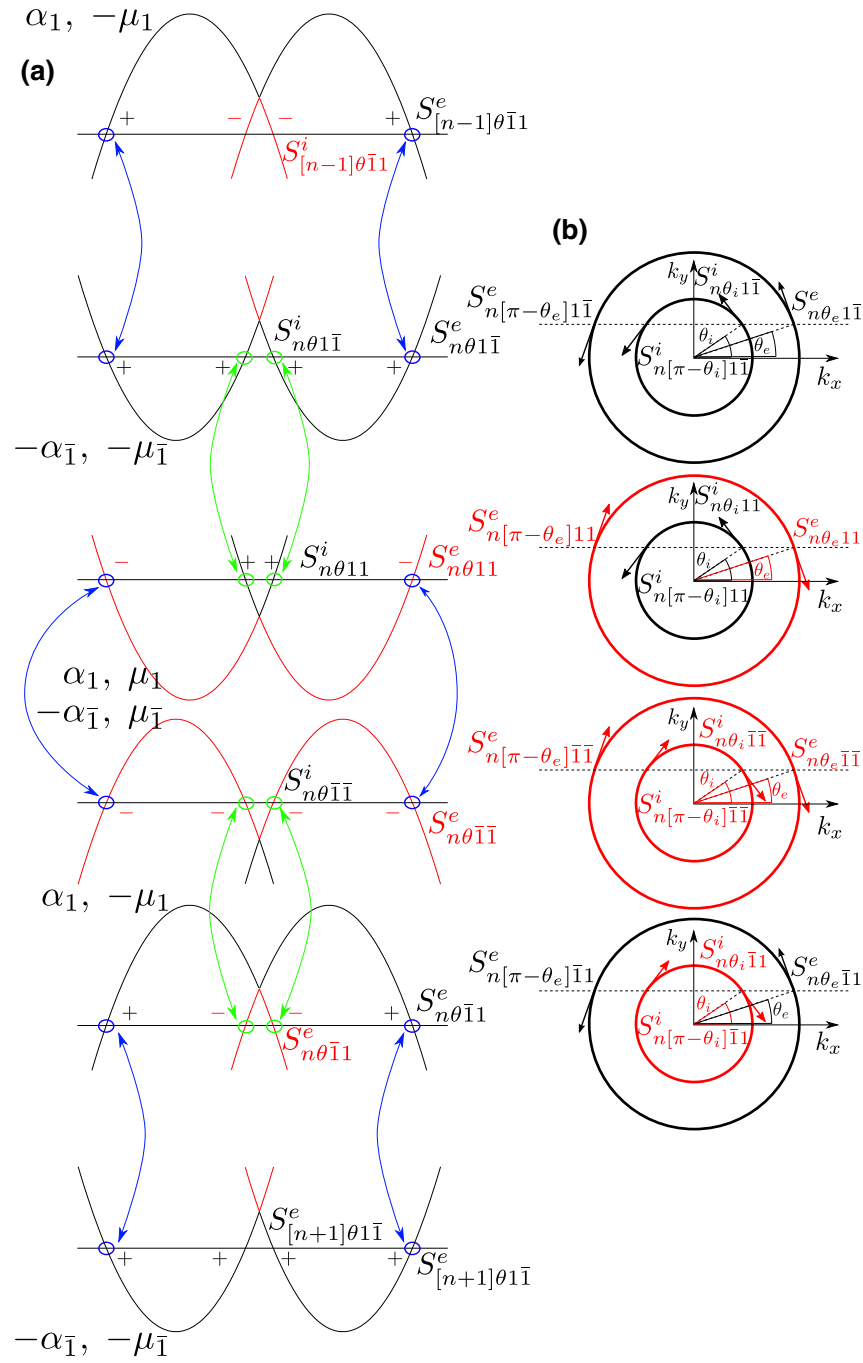

FIG. 2. (a) Dispersion relation of each layer for fixed $\theta$. The chemical potentials $\mu_{\tau}$ are chosen such that inner and outer Fermi surfaces have the same radii across different layers. The arrows indicate where the tunneling between the layers opens up gaps (small green circles). Note that the bottom and top layers stay gapless and have a dispersion consisting of a single Dirac cone with spin locked to momentum due to time reversal invariance. (b) Interior and exterior Fermi surfaces of each layer with the cuts for $k_{y}=$ const. The fields for interior (exterior) left and right movers $S_{n \theta_{i} \eta \tau}^{i}, S_{n\left[\pi-\theta_{i}\right] \eta \tau}^{i}$ $\left(S_{n \theta_{e} \eta \tau}^{e}, S_{n\left[\pi-\theta_{e}\right] \eta \tau}^{e}\right)$ have in general different spin orientations.

First, we demonstrate that the top and bottom layers host gapless modes with a helical Dirac spectrum. For the moment, we assume that the system is infinite and translationally invariant in $x$ and $y$ directions and we introduce momenta $k_{x}$ and $k_{y}$, which are good quantum numbers. Alternatively, due to rotation invariance, one can change to polar coordinates with momenta $k_{r}$ and $k_{\theta}$. This allows us to treat the problem as effectively one dimensional if the orbital degree of freedom is integrated out, see Fig. 2. The wave function can be represented close to the Fermi surface in terms of slowly varying fields $S_{n \theta \eta \tau}^{e / i}$,

$$
\Psi_{n \theta \eta \tau \sigma}(x, y)=\sum_{\delta=e, i} \alpha_{\delta \theta \eta \tau \sigma} S_{n \theta \eta \tau}^{\delta} e^{i k_{F \delta}(x \cos \theta+y \sin \theta)},
$$


with the angle $\theta \in[0,2 \pi), \alpha_{e \theta \eta \tau \sigma}=\langle\sigma \mid-\tau \cdot \eta, \theta\rangle$ and $\alpha_{i \theta \eta \tau \sigma}=\langle\sigma \mid \eta, \theta\rangle$. The kinetic term can be rewritten as

$$
\tilde{H}_{0}=-i \hbar \sum_{\substack{\delta=i, e \\ \theta \theta ; \tau, \eta= \pm 1}} \beta_{\delta} \eta v_{F \tau}\left(S_{n \theta \eta \tau}^{\delta}\right)^{\dagger} \frac{\partial}{\partial r} S_{n \theta \eta \tau}^{\delta},
$$

where $\beta_{e}=1$ and $\beta_{i}=\tau$. We also take into account that the Fermi velocities $\hbar v_{F \tau}=\partial E_{\tau} /\left.\partial k\right|_{\mu_{\tau}}$ are different. The tunneling terms induce couplings between interior/exterior Fermi surfaces of different layers,

$$
\begin{aligned}
\tilde{H}_{t}= & t \sum_{n \theta}\left[\left(S_{n \theta 11}^{e}\right)^{\dagger} S_{n \theta \overline{1} \overline{1}}^{e}+\left(S_{[n-1] \theta \overline{1} 1}^{e}\right)^{\dagger} S_{n \theta 1 \overline{1}}^{e}\right. \\
& \left.+\left(S_{n \theta 1 \overline{1}}^{i}\right)^{\dagger} S_{n \theta 11}^{i}+\left(S_{n \theta \overline{1} \overline{1}}^{i}\right)^{\dagger} S_{n \theta \overline{1} 1}^{i}\right]+ \text { H.c. }
\end{aligned}
$$

Here, we keep only nonoscillating terms and take into account the spin conservation during the tunneling, see Fig. 2(a). Importantly, all coupling terms in Eq. (8) involve fields with opposite signs of Fermi velocities and each field, except for the ones belonging to the top and bottom layers, has a partner to which it is coupled. This results in the opening of gaps at the Fermi level such that the bulk spectrum is fully gapped. However, the exterior Fermi surface field $S_{1 \theta 1 \overline{1}}^{e}$ of the top-most layer and the interior Fermi surface field $S_{N \theta \overline{1} 1}^{i}$ of the bottom-most layer do not have partners in Eq. (8) and, thus, stay gapless as all the remaining layers are insulating. As was noted above, $S_{1 \theta 1 \overline{1}}^{e}$ and $S_{N \theta \overline{1} 1}^{i}$ describe the helical Dirac cones in which spin direction is locked to the momentum direction. In our case, the spin direction stays always perpendicular to the momentum, see Fig. 2(b). Such surface states are the hallmark of a strong $3 \mathrm{D}$ TI [2]. The same Hamiltonian $\tilde{H}_{t}$ [Eq. (8)] also occurs in systems with a gradient of SOI from layer to layer (analogous to the 2D TI in Ref. [17]) and with either exclusively electron or exclusively hole spectrum in all layers.

Since the rotational symmetry is broken, it is far from obvious that the surface states exist on any 2D boundary. To this end, we demonstrate that helical surface states also exist if a hard-wall boundary is added, say, at the plane $x=0$. To this end we assume that the system is infinite in $y$ and $z$ directions. Since the system is translation invariant in the $y$ direction ( $z$ direction), $k_{y}\left(k_{z}\right)$ is a good quantum number defined via $\Psi_{k_{z}}=\Sigma_{n} e^{i n k_{z} a} \Psi_{n} / \sqrt{N}$, where $a=4 a_{z}$ is the unit-cell size. The $y$ dependence of the total wave function is given trivially as $\Psi_{k_{y} k_{z} \eta \tau \sigma}(x, y)=e^{i k_{y} y} \Psi_{k_{y} k_{z} \eta \tau \sigma}(x)$. Since both $k_{y}$ and $k_{z}$ are good quantum numbers the problem is effectively one dimensional, see Fig. 2(b). To simplify the problem further, we linearize the motion in the $x$ direction which is achieved with the ansatz following from Eq. (6),

$$
\Psi_{k_{y} k_{z} \eta \tau \sigma}(x)=\sum_{\substack{\delta=i, e \\ \theta \in\left\{\theta_{\delta}, \pi-\theta_{\delta}\right\}}} \alpha_{\delta \theta \eta \tau \sigma} S_{k_{z} \theta \eta \tau}^{\delta}(x) e^{i k_{F \delta} x \cos \theta},
$$

where $S_{k_{z} \theta \eta \tau}^{\delta}$ is the Fourier transform of $S_{n \theta \eta \tau}^{\delta}$. The above ansatz [33-35] is valid for $k_{y}<k_{F i}$ and $t \ll \mid E_{\mathrm{so}, \tau}-\hbar^{2}\left(k_{y}-\right.$ $\left.\tau k_{\text {so }, \bar{\tau}}\right)^{2} / 2 m \mid$ with $E_{y}=\hbar^{2} k_{y}^{2} / 2 m_{0}$. The angles $\theta_{i}$ and $\theta_{e}$ are defined in Fig. 2(b) or explicitly expressed by $\cos \theta_{\delta}=$ $\sqrt{k_{F \delta}^{2}-k_{y}^{2}} / k_{F \delta}$. The spin orientation is determined by $\alpha_{\delta \theta \eta \tau \sigma}$ and depends on $\theta_{\delta}$ which in turn depends on $k_{y}$, see Fig. 2(b).
After performing the above linearization [33-35], we arrive at the effective Hamiltonian

$$
\begin{aligned}
\bar{H}_{0}= & -i \sum_{\delta=i, e} \hbar \sum_{\substack{\left.\eta, \tau= \pm 1 \\
\theta \in \theta_{\delta}, \pi-\theta_{\delta}\right\}}} \beta_{\delta} \eta v_{F \tau} \cos \theta\left(S_{k_{z} \theta \eta \tau}^{\delta}\right)^{\dagger} \partial_{x} S_{k_{z} \theta \eta \tau}^{\delta} \\
\bar{H}_{t}= & t \sum_{\theta \in\left\{\theta_{i}, \pi-\theta_{i}\right\}}\left[\left(S_{k_{z} \theta 1 \overline{1}}^{i}\right)^{\dagger} S_{k_{z} \theta_{i} 11}^{i}+\left(S_{k_{z} \theta \overline{1} \overline{1}}^{i}\right)^{\dagger} S_{k_{z} \theta_{i} \overline{1} 1}^{i}\right] \\
& +t \sum_{\theta \in\left\{\theta_{e}, \pi-\theta_{e}\right\}}\left[\left(S_{k_{z} \theta 11}^{e}\right)^{\dagger} S_{k_{z} \theta \overline{1} \overline{1}}^{e}+e^{i k_{z} a}\left(S_{k_{z} \theta_{e} \overline{1} 1}^{e}\right)^{\dagger} S_{k_{z} \theta_{e} 1 \overline{1}}^{e}\right] \\
& + \text { H.c. }
\end{aligned}
$$

It is readily noticeable from Fig. 2(a) that the Hamiltonian breaks down into $2 \times 2$ blocks, formed by the fields coupled by the tunneling. After inserting the ansatz $S_{k_{z} \theta \eta \tau}^{\delta}(x) \sim e^{i k_{\delta} x}$, we arrive at the bulk spectrum around the interior and exterior Fermi surfaces,

$$
\begin{aligned}
E_{\delta, \pm}= & k_{\delta}\left(v_{1}-v_{\overline{1}}\right) \cos \theta_{\delta} \\
& \pm \sqrt{4 t^{2}+k_{\delta}^{2}\left(v_{1}+v_{\overline{1}}\right)^{2} \cos ^{2} \theta_{\delta}},
\end{aligned}
$$

where $k_{\delta}=k_{x}-k_{F \delta} \cos \theta_{\delta}$ and $\delta=e, i$. The bulk spectral gap is given by $\Delta=2 t \sqrt{v_{1} v_{\overline{1}}} /\left(v_{1}+v_{\overline{1}}\right)$. The dispersion relation is determined by

$$
\sin (2 \Omega)= \pm \frac{2 \sin \left(k_{z} a / 2\right) \cos \theta_{e} \cos \theta_{i}}{\cos \theta_{e}+\cos \theta_{i}},
$$

and plotted in the Appendix A. We note that $E\left(k_{y}, k_{z}=0\right)$ is independent of $k_{y}$, which results in degeneracy. This degeneracy is due to fact that we only retained resonant processes in our perturbation analysis [36]. If the problem is solved numerically (see below), this accidental degeneracy is lifted except at $\boldsymbol{k}=0$, where it is protected by time-reversal symmetry. Also any perturbation in the chemical potentials lifts such a degeneracy and one is left with a single anisotropic Dirac cone. To demonstrate this explicitly, we assume a detuning $\delta \mu$ of chemical potential in the first layer. For each value of $k_{y}$ there is a twofold degeneracy which is lifted by such a perturbation. After performing the perturbation expansion within the twofold degenerate subspace we arrive at the following dispersion relation:

$$
E\left(k_{y}, k_{z}=0\right)=\frac{\delta \mu}{8}\left(1-\frac{k_{F i}}{k_{F e}}\right) \frac{k_{y}}{k_{F i}},
$$

where we assumed $v_{1}=v_{\overline{1}}=v, k_{y} \ll k_{F i}$, and $t \ll k_{F i} v$, see Appendix B for details. The system is a strong 3D TI of the type $1(000)$.

We finally address the above model numerically and study the edge states along the $y z$ layer in the tight-binding model framework with $k_{y}$ and $k_{z}$ being good quantum numbers. The corresponding tight-binding Hamiltonian is given by $H=\sum_{k_{y} k_{z} \eta \tau} H_{0 k_{y} k_{z} \eta \tau}+\sum_{k_{y} k_{z}} H_{t k_{y} k_{z}}$ with

$$
\begin{aligned}
H_{0 k_{y} k_{z} \eta \tau}= & -\sum_{n \sigma}\left\{\eta\left[t_{0} \cos \left(k_{y} a_{y}\right)+\mu_{\tau} / 2\right] c_{k_{y} k_{z} \eta \tau n \sigma}^{\dagger} c_{k_{y} k_{z} \eta \tau n \sigma}\right. \\
& \left.-\eta t_{0} c_{k_{y} k_{z} \eta \tau(n+1) \sigma}^{\dagger} c_{k_{y} k_{z} \eta \tau n \sigma}\right\}
\end{aligned}
$$




$$
\begin{aligned}
& +\bar{\alpha}_{\tau} \sum_{n}\left[c_{k_{y} k_{z} \eta \tau(n-1) \overline{1}}^{\dagger} c_{k_{y} k_{z} \eta \tau n 1}-c_{k_{y} k_{z} \eta \tau(n+1) \overline{1}}^{\dagger} c_{k_{y} k_{z} \eta \tau n 1}\right. \\
& \left.+2 i \sin \left(k_{y} a_{y}\right) c_{k_{y} k_{z} \eta \tau n \overline{1}}^{\dagger} c_{k_{y} k_{z} \eta \tau n 1}\right]+ \text { H.c. } \\
H_{t k_{y} k_{z}}= & t \sum_{n \sigma\left\langle\tau \eta ; \tau^{\prime} \eta^{\prime}\right\rangle} e^{i \phi_{\tau \eta \tau^{\prime} \eta^{\prime}}} c_{k_{y} k_{z} \eta^{\prime} \tau^{\prime} n \sigma}^{\dagger} c_{k_{y} k_{z} \eta \tau n \sigma}
\end{aligned}
$$

where again the last sum runs over neighboring layers and $\bar{\alpha}_{\tau}$ is the spin-flip hopping amplitude, related to the physical SOI parameter by $\bar{\alpha}_{\tau}=\alpha_{\tau} / 2 a_{y}$ (assuming $a_{x}=a_{y}$ ) and to the SOI energy by $\bar{E}_{\mathrm{so}, \tau}=\bar{\alpha}_{\tau}^{2} / t_{0}$ [37-39]. Here, $\phi_{\overline{1} 11 \overline{1}}=$ $-\phi_{1 \overline{1} \overline{1} 1}=k_{z} a_{z}$, otherwise, $\phi_{\tau \eta \tau^{\prime} \eta^{\prime}}=0$. The lattice constant in the $i$ direction is $a_{i}$ with $i=x, y, z$. The operator $c_{k_{y} k_{z} \eta \tau n \sigma}^{\dagger}$ is an annihilation operator acting on the electron with momentum $k_{y}\left(k_{z}\right)$ in the $y(z)$ direction and with spin $\sigma$ located at the point $x=n a_{x}$ along the $x$ direction of the $\eta \tau$ layer. Our numerical results confirm the strong TI phase of the type 1(000), see Fig. 3 and Appendix C. We again observe the single anisotropic Dirac

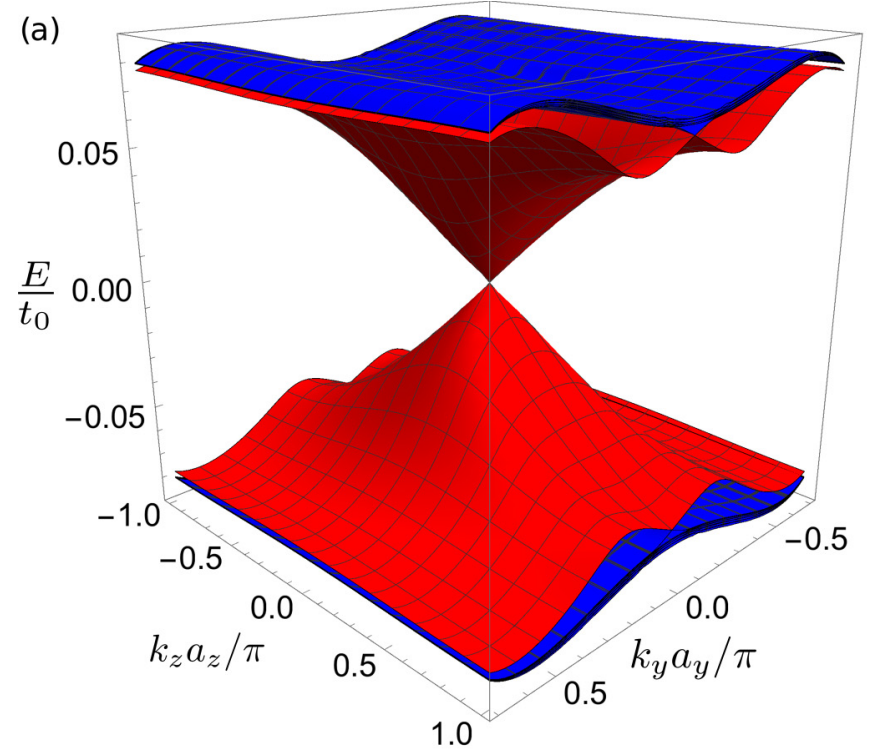

(b)

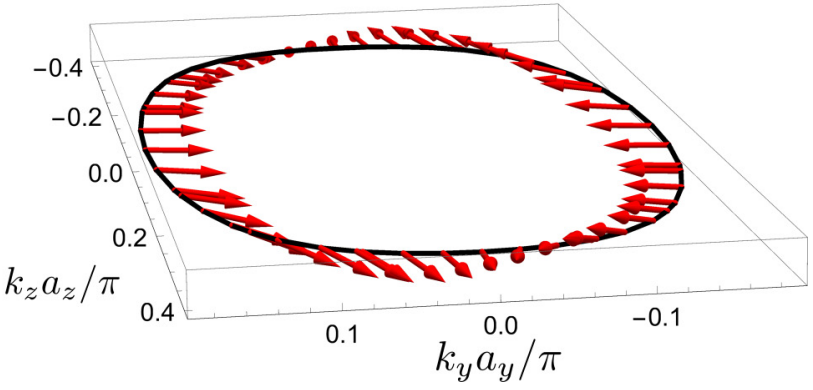

FIG. 3. (a) Dispersion relation of the surface states (red) localized in the $y z$ plane as well as bulk states (blue) obtained numerically, see Eq. (15). At small momenta, the surface states form a single anisotropic Dirac cone, but merge with the bulk states at large momenta. b) Spin orientation (red arrows) of the first layer of the unit cell for a fixed energy $E / t_{0}=-0.03$ and at the position $x_{0}=3 a_{x}$ away from the left edge. The parameter values assumed are $\bar{\alpha}_{1}=0.3 t_{0}, \bar{\alpha}_{\overline{1}}=0.55 t_{0},\left|\mu_{1}\right|=\left|\mu_{\overline{1}}\right|=0.2125 t_{0}$, and $t=0.1 t_{0}$. The spin orientation is locked to the momentum direction, confirming the strong TI phase. cone, where the accidental degeneracy at $k_{z}=0$ described before is lifted by a slight detuning of the chemical potential or due to higher-order tunneling terms not taken into account in the linearized approximation.

\section{CONCLUSIONS}

We introduced a coupled-layer approach to construct a strong 3D TI, where the building blocks are nontopological Rashba 2DEG layers. We showed that the bulk spectrum becomes gapped, with the gap being proportional to the tunnel coupling $t$ between the layers - a parameter that can be experimentally tuned. Additionally, any $2 \mathrm{D}$ boundary hosts gapless helical surface states. We calculated the dispersion relation of these surface states and found a single Dirac cone at $\boldsymbol{k}=0$, which together with the bulk gap constitutes a hallmark of a strong 3D TI [2].

\section{ACKNOWLEDGMENTS}

We acknowledge support from the Swiss NSF and NCCR QSIT.

\section{APPENDIX A: DETAILS OF THE ANALYTICAL CALCULATION}

To obtain the spectrum of the surface states we fix the parameters (including the energy inside the gap) and find the eight decaying eigenstates of the Hamiltonian. Using Eq. (9), we express them on the basis of the original fermionic fields $\Psi(x)$, leading to eight eight-spinor solutions $\Phi^{j}(x)$ with $j=$ $1, \ldots, 8$, and construct an $8 \times 8$ Wronskian matrix $W_{i j}(x)=$ $\left[\Phi^{j}(x)\right]_{i}$. The equation det $W(0)=0$ gives the spectrum of the surface states [36]. We note that for $k_{y} \neq 0$, the interior and exterior branches have different velocities in the $x$ direction. After substituting $E=\Delta \cos \Omega$ with $\Omega \in[0, \pi]$ and assuming $\theta_{e}, \theta_{i} \in[0, \pi / 2)$ we obtain

$$
\begin{aligned}
\operatorname{det} W(0)= & \frac{v_{2} e^{i k_{z} a / 2}}{v_{1}}\left[4 \sin ^{2}\left(k_{z} a / 2\right) \cos ^{2} \theta_{e} \cos ^{2} \theta_{i}\right. \\
& \left.-\left(\cos \theta_{e}+\cos \theta_{i}\right)^{2} \sin ^{2}(2 \Omega)\right] .
\end{aligned}
$$

Thus, the dispersion from Eq. (13) in the main text, shown in Fig. 4, is obtained.

\section{APPENDIX B: DETUNING OF THE CHEMICAL POTENTIAL}

In this Appendix we show that any perturbation of the chemical potential in one of the layers lifts the degeneracy for $k_{z}=0$ which is depicted in Fig. 4. To demonstrate this explicitly we assume a detuning $\delta \mu$ of chemical potential in the first layer. For each value of $k_{y}$ there is twofold degeneracy which is lifted by such a perturbation. After performing the perturbation expansion in lowest order within the twofold degenerate subspace we arrive at the following dispersion relation:

$$
E\left(k_{y}, k_{z}=0\right)=\delta \mu f\left(\frac{k_{F e}}{k_{F i}}, \frac{t}{k_{F i} v}\right) \frac{k_{y}}{k_{F i}},
$$




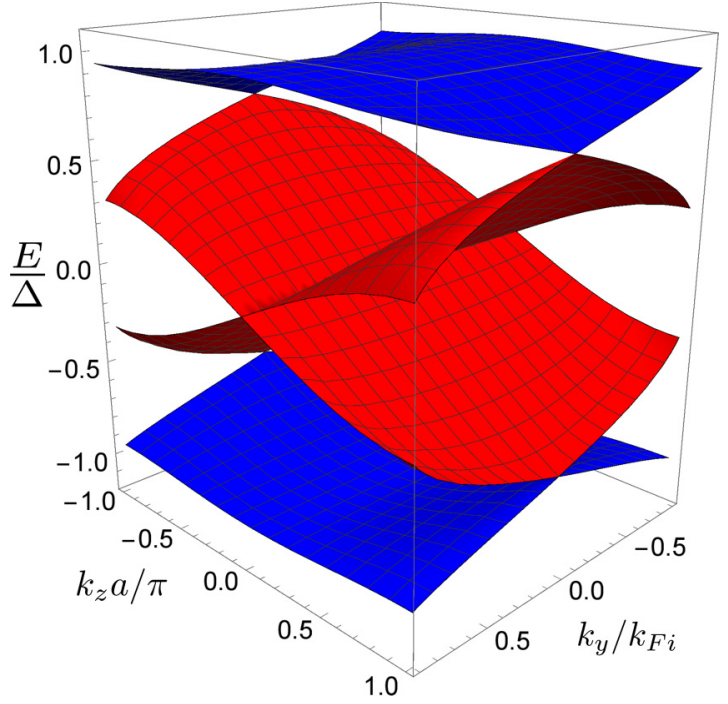

FIG. 4. Dispersion relation of the surface states localized in the $y z$ plane for $k_{F e} / k_{F i}=3$, obtained analytically from Eq. (13) of the main text with $E / \Delta=\cos \Omega$. We plot $k_{y}$ up to value of $0.9 k_{F i}$, the concrete range of validity of the dispersion depends on the value of $t$ and is given below Eq. (9).

where we assumed $v_{1}=v_{\overline{1}}=v$ and $k_{y} \ll k_{F i}$. The function $f(x, y)$ is given by $(x>1)$

$$
\begin{aligned}
f(x, y)= & \frac{\left(x^{2}-1\right)^{2}}{8 x\left[\left(x^{2}-1\right)^{2}+4 y\left(x^{2}+1\right)\right]} \sqrt{1+\frac{4 y^{2}}{(x-1)^{2}}} \\
& \times \sqrt{\left[2 y(x-1)^{2}-4 y^{3}\right]^{2}+\left(x+6 y^{2}\right)^{2}} .
\end{aligned}
$$

Since our analysis is valid for $t \ll k_{F i} v$ [we took only resonant terms into account in Eq. (5)] we can further simplify the above dispersion by expanding for small $t /\left(k_{F i} v\right)$ and arrive at Eq. (14).

\section{APPENDIX C: NUMERICAL CALCULATION OF 2D SURFACE STATES SPECTRUM}

In this Appendix we compare our numerical results to analytical ones and additionally give more details about the numerical results. Our analytical results are valid for $t \ll\left|\mu_{\tau}\right|, E_{\mathrm{so}, \tau}$, and in this limit we obtain the degeneracy for $k_{z}=0$, see Fig. 4. This degeneracy is lifted linearly in $k_{y}$ for $k_{y} \ll k_{F i}$ as shown in the main text. Our numerical tight-binding simulation confirms all these features, see Fig. 5. Namely, around $k_{y}=0$ the degeneracy is linearly lifted since for the tight-binding model it is very difficult to tune the sizes of the Fermi surfaces to be the same across the layers. Additionally, we find that there is a remaining degeneracy at $k_{y} \sim k_{F i}\left(k_{F i} \sim 0.2 \pi / a_{y}\right.$ for the parameters in Fig. 5).

We found that increasing the tunnel coupling between the layers, above the limit where the linearization works $t \sim E_{\mathrm{so}, 1}$, the $k_{y}=0$ degeneracy gets completely lifted and one obtains a single Dirac cone at $k_{y}=0$, see Fig. 3. Additionally, in

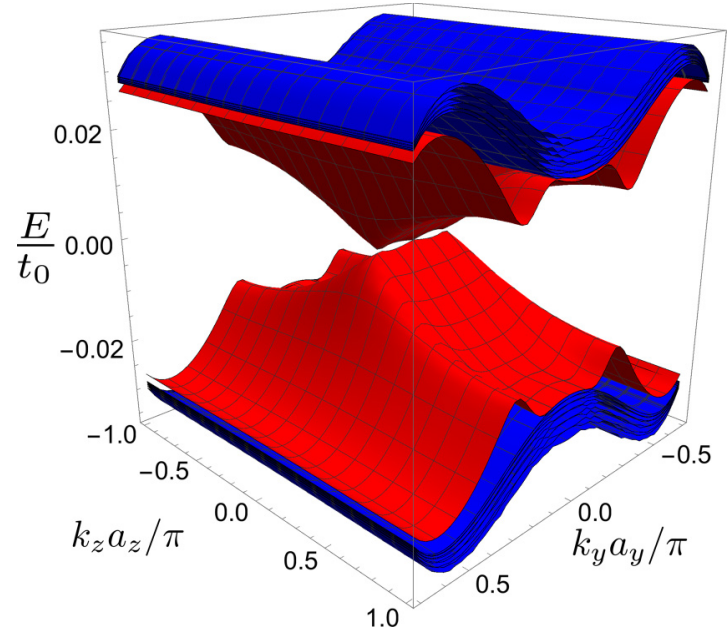

FIG. 5. Dispersion relation of the surface states localized in the $y z$ plane obtained numerically in the tight-binding model. The parameters of the system take the following values: $\bar{\alpha}_{1}=0.3 t_{0}$, $\bar{\alpha}_{\overline{1}}=0.55 t_{0},\left|\mu_{1}\right|=\left|\mu_{\overline{1}}\right|=0.2125 t_{0}$, and $t=0.05 t_{0}$.

Fig. 6(a) [Fig. 6(b)] we plot the cuts $k_{z}=0\left[k_{y}=0\right]$ of the dispersion relation which show that there is no additional structure inside of the Dirac cone. Figure 6(a) shows the behavior of the surface states within the whole Brillouin zone from where it is seen that the dispersion relation curve of the surface state does not bend down.
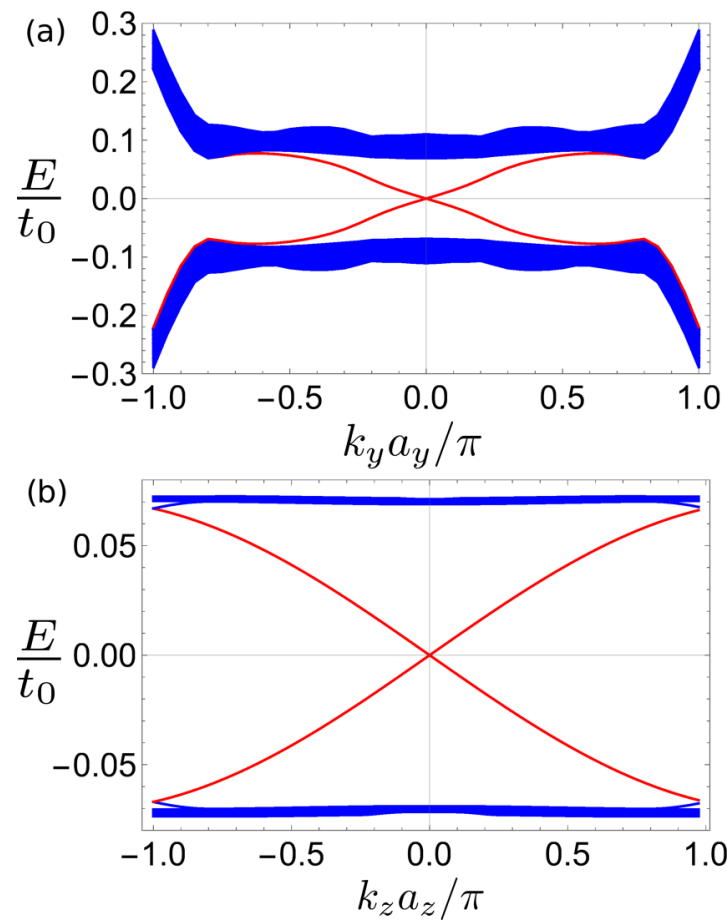

FIG. 6. The (a) $k_{z}=0$ and (b) $k_{y}=0$ cuts of the dispersion relation of the surface states localized in the $y z$ plane obtained numerically in the tight-binding model. The parameters of the system take the following values: $\bar{\alpha}_{1}=0.3 t_{0}, \bar{\alpha}_{\overline{1}}=0.55 t_{0},\left|\mu_{1}\right|=\left|\mu_{\overline{1}}\right|=$ $0.2125 t_{0}$, and $t=0.1 t_{0}$. 
[1] K. v. Klitzing, G. Dorda, and M. Pepper, Phys. Rev. Lett. 45, 494 (1980).

[2] M. Z. Hasan and C. L. Kane, Rev. Mod. Phys. 82, 3045 (2010).

[3] A. P. Schnyder, S. Ryu, A. Furusaki, and A. W. W. Ludwig, Phys. Rev. B 78, 195125 (2008).

[4] A. Kitaev, Ann. Phys. 303, 2 (2003).

[5] B. Volkov and O. Pankratov, JETP Lett. 42, 145 (1985).

[6] K. Nomura, M. Koshino, and S. Ryu, Phys. Rev. Lett. 99, 146806 (2007).

[7] D. Poilblanc, G. Montambaux, M. Héritier, and P. Lederer, Phys. Rev. Lett. 58, 270 (1987).

[8] L. P. Gor'kov and A. G. Lebed, Phys. Rev. B 51, 3285 (1995).

[9] C. L. Kane, R. Mukhopadhyay, and T. C. Lubensky, Phys. Rev. Lett. 88, 036401 (2002).

[10] J. Klinovaja and D. Loss, Phys. Rev. Lett. 111, 196401 (2013).

[11] J. C. Y. Teo and C. L. Kane, Phys. Rev. B 89, 085101 (2014).

[12] J. Klinovaja and D. Loss, Eur. Phys. J. B 87, 171 (2014).

[13] T. Meng, P. Stano, J. Klinovaja, and D. Loss, Eur. Phys. J. B 87, 203 (2014).

[14] J. Klinovaja and Y. Tserkovnyak, Phys. Rev. B 90, 115426 (2014).

[15] T. Neupert, C. Chamon, C. Mudry, and R. Thomale, Phys. Rev. B 90, 205101 (2014).

[16] E. Sagi and Y. Oreg, Phys. Rev. B 90, 201102 (2014).

[17] J. Klinovaja, Y. Tserkovnyak, and D. Loss, Phys. Rev. B 91, 085426 (2015).

[18] R. A. Santos, C.-W. Huang, Y. Gefen, and D. B. Gutman, Phys. Rev. B 91, 205141 (2015).

[19] E. Sagi, Y. Oreg, A. Stern, and B. I. Halperin, Phys. Rev. B 91, 245144 (2015).

[20] E. M. Stoudenmire, D. J. Clarke, R. S. K. Mong, and J. Alicea, Phys. Rev. B 91, 235112 (2015).
[21] S. Sahoo, Z. Zhang, and J. C. Y. Teo, arXiv:1509.07133.

[22] T. Meng, Phys. Rev. B 92, 115152 (2015).

[23] E. Sagi and Y. Oreg, . Phys. Rev. B 92, 195137 (2015)

[24] Y. A. Bychkov and E. I. Rashba, JETP Lett. 39, 78 (1984).

[25] R. A. Deutschmann, W. Wegscheider, M. Rother, M. Bichler, G. Abstreiter, C. Albrecht, and J. H. Smet, Phys. Rev. Lett. 86, 1857 (2001).

[26] B. Sachs, L. Britnell, T. O. Wehling, A. Eckmann, R. Jalil, B. D. Belle, A. I. Lichtenstein, M. I. Katsnelson, and K. S. Novoselov, App. Phys. Lett. 103251607 (2013).

[27] K. Novoselov, V. Fal'ko, L. Colombo, P. Gellert, M. Schwab, and K. Kim, Nature 490, 192 (2012).

[28] R. A. Żak, D. L. Maslov, and D. Loss, Phys. Rev. B 82, 115415 (2010).

[29] A. Bermudez, L. Mazza, M. Rizzi, N. Goldman, M. Lewenstein, and M. A. Martin-Delgado, Phys. Rev. Lett. 105, 190404 (2010).

[30] S. Nakosai, Y. Tanaka, and N. Nagaosa, Phys. Rev. Lett. 108, 147003 (2012).

[31] E. Bernardes, J. Schliemann, M. Lee, J. C. Egues, and D. Loss, Phys. Rev. Lett. 99, 076603 (2007).

[32] A. Ohtomo and H. Y. Hwang, Nature 427, 423 (2004).

[33] B. Braunecker, G. I. Japaridze, J. Klinovaja, and D. Loss, Phys. Rev. B 82, 045127 (2010).

[34] J. Klinovaja and D. Loss, Phys. Rev. B 86, 085408 (2012).

[35] J. Klinovaja and D. Loss, Phys. Rev. Lett. 110, 126402 (2013).

[36] J. Klinovaja, P. Stano, and D. Loss, Phys. Rev. Lett. 109, 236801 (2012).

[37] D. Chevallier, D. Sticlet, P. Simon, and C. Bena, Phys. Rev. B 85, 235307 (2012).

[38] D. Rainis, L. Trifunovic, J. Klinovaja, and D. Loss, Phys. Rev. B 87, 024515 (2013).

[39] J. Klinovaja and D. Loss, Eur. Phys. J. B 88, 62 (2015). 